\title{
Ca-Operative Bird
}

Compiled by

Thirteen Saskatchewan observers contributed spring migration dates (for half or more of the 29 species) to this study in co-operation with the Wisconsin Society of Ornithology and the U.S. Fish and Wildlife Service. Unfortunately

\begin{tabular}{|c|c|c|c|c|c|c|}
\hline Canada Goose ..... & Apl3 & Apl 2 & Ap8 & My5 & Ap10 & Ap8 \\
\hline 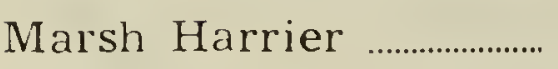 & $\operatorname{Mr} 7$ & $\operatorname{Mr} 28$ & $\operatorname{Mr} 22$ & $\operatorname{Mr} 18$ & Ap4 & Ap21 \\
\hline 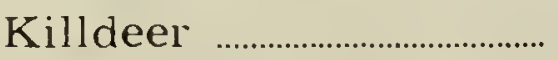 & Apr 12 & - & Ap11 & A p12 & Ap 13 & Ap13 \\
\hline ourning Dove.. & My9 & Ap27 & Ap 16 & My 6 & - & Ap21 \\
\hline ghthawk ......... & - & - & $\operatorname{Jn} 6$ & Jn1 & My 24 & Jn3 \\
\hline T. Hummingbird .... & - & 一 & Jn11 & $\operatorname{Jn} 12$ & $\operatorname{Jn} 20$ & Jn6 \\
\hline S. Flicker & A p20 & Ap26 & Ap24 & Ap 23 & Ap24 & A p27 \\
\hline 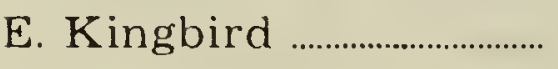 & My 17 & My 20 & My 18 & My 14 & My21 & My 21 \\
\hline 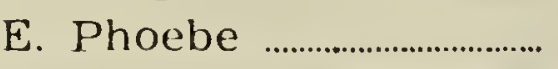 & - & - & Ap27 & - & - & My 15 \\
\hline arn Swallow ..... & My 10 & My 12 & My13 & My 10 & My 12 & My 13 \\
\hline arple Martin ... & My21 & - & - & Jn6 & - & - \\
\hline OW & $\operatorname{Mr} 20$ & Mr21 & Mr30 & $\operatorname{Mr} 22$ & Mr20 & Mr7 \\
\hline 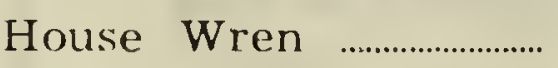 & $\mathrm{Jn} 2$ & My22 & My 8 & My 21 & My21 & My 19 \\
\hline atbird & Jn 12 & My 17 & My 20 & My24 & $\operatorname{Jn} 8$ & My 26 \\
\hline 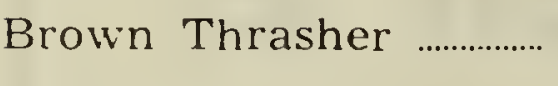 & My21 & My 20 & My 17 & My20 & My 20 & - \\
\hline d-Eyed Vireo & - & 一 & My23 & - & 一 & Jn3 \\
\hline ack and White W....... & 一 & - & My 16 & - & - & My 14 \\
\hline llow Warbler & My 13 & My22 & My 12 & My 20 & My31 & My 15 \\
\hline 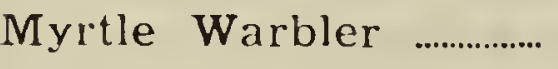 & Ap24 & - & Ap28 & My 16 & My22 & Ap26 \\
\hline 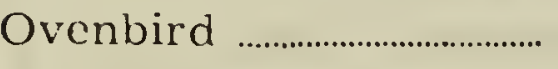 & - & & My 23 & - & - & My 27 \\
\hline istart ............ & My 23 & 一 & My 23 & - & 一 & My23 \\
\hline d-W. Blackbird ............ & Apl 6 & Ap 19 & Ap16 & Ap20 & My 14 & My 6 \\
\hline litimore Oriole & My 21 & Ap22 & My21 & My 27 & My24 & My21 \\
\hline se-Br. Grosbeak ...... & - & - & My 18 & My29 & - & My 14 \\
\hline ldfinch & My25 & My22 & My 21 & My24 & My31 & My 27 \\
\hline te-Col. Junco ..... & Ap9 & Mr 15 & Ap 10 & Ap5 & Ap9 & Ap13 \\
\hline ipping Sparrow .......... & Ap26 & - & My6 & My 16 & My 12 & My 12 \\
\hline ite-Crwn. Sparrow... & My2 & My 19 & My 7 & My8 & - & My 14 \\
\hline nite-Thr. Sparrow ...... & - & - & Myl & My9 & - & My 12 \\
\hline
\end{tabular}




\section{Migration Study 1954}

\section{DR. STUART HOUSTON}

again this year our reports are concentrated in the eastern half of the province.

It is of interest that some species, such as the Killdeer and Redstart, seem to arrive almost simultaneously at all points.

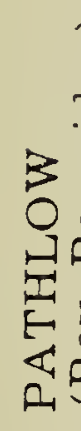

\begin{tabular}{|c|c|c|c|c|c|c|c|c|}
\hline Ap 21 & Ap8 & Ap8 & Ap 14 & My8 & My 16 & Ap 8 & Ap 12 & Ap 12 \\
\hline Ap 13 & Ap9 & Apl & Ap 14 & - & Apl 6 & Ap 18 & Apl4 & Apl2 \\
\hline Ap21 & Ap 12 & Ap 12 & Ap 12 & My3 & Apl 6 & Ap 11 & Ap 16 & Ap 13 \\
\hline My 7 & My 10 & Ap 19 & My4 & 一 & My 12 & Ap14 & - & My 9 \\
\hline Jn 14 & My31 & My 25 & Jn4 & Jn3 & My24 & My24 & Jn6 & - \\
\hline Jn10 & Jn 11 & My 20 & Jn3 & $\mathrm{Jn} 2$ & $\operatorname{Jn} 5$ & $\operatorname{Jn} 13$ & Jn10 & - \\
\hline My 1 & Ap24 & Mr3 & Ap26 & My5 & My9 & Ap25 & My30 & My6 \\
\hline My27 & My 21 & My 13 & My21 & My24 & Jn4 & Miy 24 & Jn6 & My28 \\
\hline My 7 & Ap21 & Ap 24 & My 13 & My4 & My 1 & My2 & My11 & My5 \\
\hline My 17 & My 12 & Ap 19 & My 12 & My 15 & - & My 12 & My27 & My19 \\
\hline My 14 & My 23 & - & - & - & - & Jn6 & - & - \\
\hline MTr30 & $\operatorname{Mr} 22$ & Mr23 & A p8 & Ap27 & Ap 10 & Mr16 & Ap3 & Ap 1 \\
\hline My20 & Мy 19 & My 17 & Му 20 & Jn 1 & My 23 & My24 & My 19 & My 19 \\
\hline My 27 & My 23 & My 18 & My26 & - & Jn6 & Jn6 & Jn6 & My28 \\
\hline- & - & My 18 & - & - & - & My20 & - & My28 \\
\hline $\operatorname{Jn} 14$ & - & Мy26 & Jn3 & - & My24 & Jn 13 & - & - \\
\hline - & My 23 & - & My 15 & - & My 16 & My 16 & My22 & - \\
\hline My22 & My 15 & My26 & My 12 & Jn6 & My 16 & My22 & My 13 & My27 \\
\hline Ap 14 & Ap25 & Ap28 & My5 & My 14 & Ap25 & Ap25 & Ap15 & 一 \\
\hline My27 & My 19 & - & - & - & My 23 & 一 & My23 & - \\
\hline My 17 & My 22 & My23 & My24 & - & - & My 25 & My 24 & - \\
\hline Ap21 & Ap20 & Ap20 & Ap20 & - & My 15 & Ap 13 & My2 & My2 \\
\hline My26 & My30 & My22 & My 20 & 一 & Jn5 & My 24 & My 25 & My23 \\
\hline My 27 & My 18 & My24 & My21 & My6 & My 23 & My24 & My25 & - \\
\hline Jn 1 & My31 & - & My27 & - & Jn4 & $\mathrm{Jn} 4$ & Jn6 & $\mathrm{Jn} 15$ \\
\hline Ap 10 & Ap7 & Ap 10 & Ap11 & Ap30 & Ap 13 & Ap7 & Ap14 & Ap 12 \\
\hline My 15 & My 16 & - & My29 & My3 & My 8 & My 9 & My 16 & My30 \\
\hline My 11 & - & Ap20 & My 15 & My3 & - & - & - & - \\
\hline My 10 & My 9 & Ap 28 & My 11 & My5 & My 10 & My9 & My 12 & - \\
\hline
\end{tabular}

